\title{
Hypercalcemia Is a Risk Factor for the Progression of Aortic Calcification in Kidney Transplant Recipients
}

\author{
Toshihide Naganuma $^{a}$ Yoshiaki Takemoto ${ }^{a}$ Junji Uchida ${ }^{a}$ \\ Tatsuya Nakatani $^{a}$ Daijiro Kabatab ${ }^{b}$ Ayumi Shintani ${ }^{b}$ \\ aDepartment of Urology, Osaka City University Graduate School of Medicine, Osaka, Japan; \\ ${ }^{b}$ Department of Medical Statistics, Osaka City University Graduate School of Medicine, \\ Osaka, Japan
}

\section{Keywords}

Agatston score · Vascular calcification · Kidney transplantation · Multi-slice computed tomography $\cdot$ Risk factor $\cdot$ Infrarenal abdominal aorta

\begin{abstract}
Background/Aims: Vascular calcification is common and progressive in chronic kidney disease, including kidney transplant recipients (KTRs). However, the risk factors associated with the progression of aortic calcification (AoC) in KTRs have not been fully elucidated. In the present study, we evaluated $\mathrm{AoC}$ and examined the factors associated with its advancement in KTRs. Materials: This was a prospective longitudinal study that included $98 \mathrm{KTRs}$. We quantitatively investigated infrarenal abdominal AoC using the Agatston score, as measured by multi-slice computed tomography. After the baseline investigation, a follow-up scan was performed after 3 years, and the Agatston scores were obtained again. The changes in laboratory data affecting the 2nd Agatston scores were examined by multivariable analysis using non-linear regression after adjustment for several confounders. Results: The 2nd Agatston scores were significantly greater than the baseline Agatston scores $(p<0.001)$. After adjustment for the confounders, the change in corrected serum calcium exhibited a significant nonlinear correlation with the 2 nd Agatston scores ( $p=0.022$ for non-linearity $/ p=0.031$ for the effect of corrected serum calcium). Moreover, an interaction was present from the baseline AoC in the effect of corrected serum calcium on the progression of AoC, and the effect of hypercalcemia was greater in patients with higher baseline Agatston scores $(p=0.049)$. Conclusion: The present study revealed that hypercalcemia is a risk factor for the development of infrarenal abdominal AoC in KTRs. Furthermore, the effect of hypercalcemia was greater in patients with more severe vascular calcification.




\section{Kidney \\ Blood Pressure \\ Research}

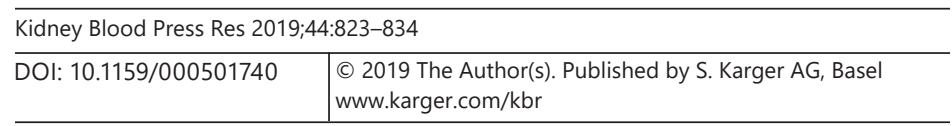

Naganuma et al.: Aortic Calcification in Kidney Transplant Recipients

\section{Introduction}

Even after successful kidney transplantation, cardiovascular disease (CVD) remains the leading cause of death among kidney transplant recipients (KTRs); with a 3.5-5\% annual risk of fatal or nonfatal CVD events compared with the general population, even after adjustment for risk factors [1, 2]. In the general population, it is well established that vascular calcification (VC) is a prognostic marker of CVD events, and cardiovascular mortality [3-6]. Moreover, VC is highly prevalent and often progressive in patients with chronic kidney disease (CKD) $[7,8]$, particularly in dialysis patients $[9,10]$, and the presence and progression of $\mathrm{VC}$ is closely associated with the increased morbidity and mortality of CVD [7-10]. There have been many reports of advanced VC in kidney transplant recipients (KTRs) [11-23], and several studies have shown that VC strongly predicts the morbidity and mortality of CVD in KTRs [12, 18, 20]. However, most of these findings were based upon observational studies in which coronary artery calcification (CAC) was evaluated quantitatively using the Agatston score [11, 13-23], whereas only a few studies have assessed aortic calcification (AoC) $[12,14,16,17]$. In this study, we performed a quantitative assessment of infrarenal abdominal AoC, using the Agatston score by multi-slice computed tomography (CT) and investigated the risk factors for the progression of VC in KTRs. In addition, the Agatston scores exhibiting greatly distorted data distribution were analyzed using non-linear regression analysis in consideration of any non-linear association.

\section{Materials and Methods}

\section{Study Design and Participants}

This was a prospective longitudinal study that included 98 KTRs. The inclusion criteria were (1) Consecutive, adult KTRs, (2) Out-patients of Osaka City University Hospital, (3) Patients who were more than 6 months post-transplant, and (4) Patients who gave informed consent prior to their participation in the study. The exclusion criteria were (1) Patients with malignancy, clinically overt infection, acute or severe illness, and (2) Patients who were pregnant or possibly pregnant. We planned to recruit participants from April 2010 to March 2014. Of the 111 patients that underwent baseline multi-slice CT, 4 cases with incomplete records were excluded, 9 cases were lost to follow-up: 6 cases died, 2 cases initiated dialysis and one transferred to another hospital, resulting in 98 cases that underwent a 2nd CT and were examined for this study. This study protocol was conducted in accordance with the Principles of the Declaration of Helsinki and the Declaration of Istanbul, and was approved by the ethics committee of the Osaka City University Graduate School of Medicine (No. 1415).

The demographic and baseline clinical data are presented in Table 1.

\section{Measurement of the Agatston Score by Multi-Slice Computed Tomography}

The protocol for multi-slice CT for measurement of Agatston score was derived from our previous study (24) and the protocol is provided in detail in the online supplementary material (see www.karger.com/doi/10.1159/000501740). We quantitatively investigated infrarenal abdominal AoC using the Agatston score, as measured by multi-slice CT from the renal artery to the bifurcation [24]. After the baseline investigation, a second multi-slice CT was performed after 3 years, and the Agatston scores were determined again.

The presence of AoC was defined by an Agatston score of $>0$. The progression of calcification was defined as the difference between the follow-up square-root transformed score (SRC) and the baseline SRC $\geq 2.5$ [25]. A calcium score $>30$ has been reported to be highly reproducible $(8-10 \%$ interscan variability) [26]. Therefore, we calculated the annualized 
Table 1. Baseline characteristics of the study subjects $(n=98)$

\begin{tabular}{|c|c|}
\hline Variables & Median (IQR) or percentage \\
\hline Age, years & $49(40,58)$ \\
\hline Sex (male), \% & 62.2 \\
\hline Body mass index & $20.7(18.9,22.5)$ \\
\hline Dialysis duration, years & $3.4(1.1,9.9)$ \\
\hline Time from transplantation, years & $5.8(2.8,10.0)$ \\
\hline Living donor, $\%$ & 79.4 \\
\hline Calcineurin inhibitor (cyclosporine), \% & 61.2 \\
\hline $\mathrm{eGFR}, \mathrm{mL} / \mathrm{min} / 1.73 \mathrm{~m}^{2}$ & $48.5(39.9,54.4)$ \\
\hline Serum creatinine, $\mathrm{mg} / \mathrm{dL}$ & $1.2(1.0,1.5)$ \\
\hline Diabetes mellitus, $\%$ & 23.5 \\
\hline $\mathrm{HbA1c}, \%$ & $6.0(5.6,6.4)$ \\
\hline Hypertension, \% & 88.8 \\
\hline Systolic blood pressure, $\mathrm{mm} \mathrm{Hg}$ & $120(113.3,130)$ \\
\hline Diastolic blood pressure, $\mathrm{mm} \mathrm{Hg}$ & $74(67.3,78.8)$ \\
\hline Dyslipidemia, $\%$ & 33.7 \\
\hline Total cholesterol, mg/dL & $197(170,221)$ \\
\hline Triglycerides, mg/dL & $104(75,132)$ \\
\hline High density lipoprotein cholesterol, mg/dL & $65(50,77)$ \\
\hline Low density lipoprotein cholesterol, mg/dL & $105.8(87.2,130.6)$ \\
\hline Serum albumin, $\mathrm{g} / \mathrm{dL}$ & $4.1(3.9,4.3)$ \\
\hline C-reactive protein, $\mathrm{mg} / \mathrm{dL}$ & $0.03(0.01,0.05)$ \\
\hline Corrected calcium, mg/dL & $10.0(9.6,10.3)$ \\
\hline Phosphate, $\mathrm{mg} / \mathrm{dL}$ & $3.0(2.6,3.3)$ \\
\hline $\mathrm{i}-\mathrm{PTH}, \mathrm{pg} / \mathrm{mL}$ & $78(61,115)$ \\
\hline Smoking habit, \% & 6.2 \\
\hline Use of statins, $\%$ & 35.1 \\
\hline Aortic calcification (Agatston score $>0$ ) & 78.6 \\
\hline
\end{tabular}

Values are expressed as the percentage for categorical variables and median (IQR) for continuous variables. IQR, interquartile range; eGFR, estimated glomerular filtration rate; HbA1c, hemoglobin A1c; i-PTH, intact parathyroid hormone.

percentage rate of progression only in those with a baseline score $>30[100 \times$ (second Agatston score - baseline Agatston score)/(baseline Agatston score) interval between scans].

\section{Definition of Traditional Risk Factors}

Subjects were admitted to our clinical research unit at 8:30 9:30 a.m. without taking immunosuppressive medications after an 8-12-h overnight fasting period. Fasting blood samples were drawn to determine the clinical data. Serum calcium was corrected by serum albumin, according to the formula: Corrected $\mathrm{Ca}[\mathrm{mg} / \mathrm{dL}]=$ measured $\mathrm{Ca}[\mathrm{mg} / \mathrm{dL}]+(4-$ serum albumin $[\mathrm{g} / \mathrm{dL}])$, when serum albumin was below $4.0 \mathrm{~g} / \mathrm{dL}$.

Hypertension (HT) was defined by (a) the administration of antihypertensive agents and/or a history of this disorder; (b) a systolic blood pressure greater than $140 \mathrm{~mm} \mathrm{Hg}$; or (c) a diastolic blood pressure greater than $90 \mathrm{~mm} \mathrm{Hg}$; blood pressure was measured prior to the outpatient consultation. Diabetes mellitus was defined as (1) administration of insulin or oral antidiabetic agents, (2) prior diagnosis according to the Report of the Expert Committee on the Diagnosis and Classification of Diabetes Mellitus of the American Diabetes Association [27]. Body mass index (BMI) was calculated by the equation [(weight in kilograms) / (square of height in meters)]. 


\section{Kidney \\ Blood Pressure \\ Research}

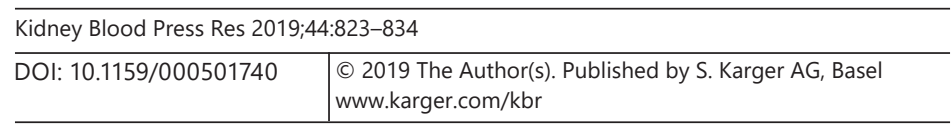

Naganuma et al.: Aortic Calcification in Kidney Transplant Recipients

\section{Statistical Analysis}

To express the patients' baseline demographical and clinical characteristics, median and interquartiles were used for continuous variables whereas percentage was used for categorical variables. Differences between the baseline and 2nd Agatston scores were examined using the Wilcoxon rank sum test.

The effects of the candidates of risk factors on the 2nd Agatston scores were assessed with multivariable linear regression models. Each risk factor was included separately in a multivariable regression model adjusting for the baseline Agatston score, age, sex, donor source, eGFR, body mass index (BMI), days after transplantation, dialysis duration, the presence of diabetes mellitus, and past history of parathyroidectomy. For the risk factors of laboratory measures (serum corrected calcium, phosphate, calcium-phosphate product, eGFR, albumin, CRP, SBP, DBP, BMI, triglyceride, HDL-cholesterol, LDL-cholesterol, iPTH, HbA1c), the change in the values (2nd measured value - 1st measured value) were used. The other risk factors include current smoking status, statin use, and use of calcium inhibitors at baseline. In order to assess the non-linearity between the laboratory variables and the baseline Agatston score, non-linear restricted-cubic-spline was conducted in the regression model.

To consider whether the effects of these risk factors on 2nd Agatston score were modified by the baseline Agatston score (interaction), we performed similar multivariable linear regression analysis described above with a cross-product term between each risk factor and the baseline Agatston score. Adjustment for the baseline characteristics and non-linearity of the laboratory variables and baseline Agatston score were considered similarly to the linear regression analysis described above.

In these analyses, missing data were imputed with the multiple imputation method using "areg.impute" function in the "rms" package of R software (https://cran.r-project.org/web/ packages/rms/rms.pdf).

All statistical inferences were made with 2-sided significance level of 5\% and conducted with R software version 3.3.1 (https://www.r-project.org/foundation/) using the "rms" package.

\section{Results}

Baseline Characteristics of the Study Subjects (Table 1)

Table 1 presents the clinical and radiological characteristics of the 98 KTRs at baseline. The median age was 49 years, and $62.2 \%$ of the participants were men. The median dialysis duration, time from transplantation and estimated glomerular filtration rate (eGFR) were 3.4, 5.8 years and $48.5 \mathrm{~mL} / \mathrm{min} / 1.73 \mathrm{~m}^{2}$, respectively. Diabetes mellitus, hypertension and dyslipidemia were found in $23.5,88.8$ and $33.7 \%$ of the participants, respectively. Of the participants, $6.2 \%$ had a smoking habit (current smoking). Of the 98 patients, AoC with an Agatston score $>0$ was detected in 77 (78.6\%), and the remaining 21 (21.4\%) exhibited no AoC (Agatston score $=0$ ).

\section{Change of the Agatston Scores (Fig. 1)}

The medians of the baseline and 2nd Agatston scores were 713.9 (12.9-3,663.7), 999.8 (69.4-4,899.9), respectively. The 2nd Agatston scores were significantly greater than the baseline Agatston scores $(p<0.001)$. At the follow-up scan, which was performed after a median $3.2(3.0-5.1)$ years, the prevalence of aortic calcification increased to $84.7 \%$ and individual Agatston scores progressed in $63.9 \%$ of patients. AoC increased by a median of 8.2 (4.0-20.8) \% per year in KTRs with baseline Agatston score $>30$. The 2 nd Agatston score was also 0 in $15(71.4 \%)$ of the 21 KTRs with a baseline Agatston score of 0 . 


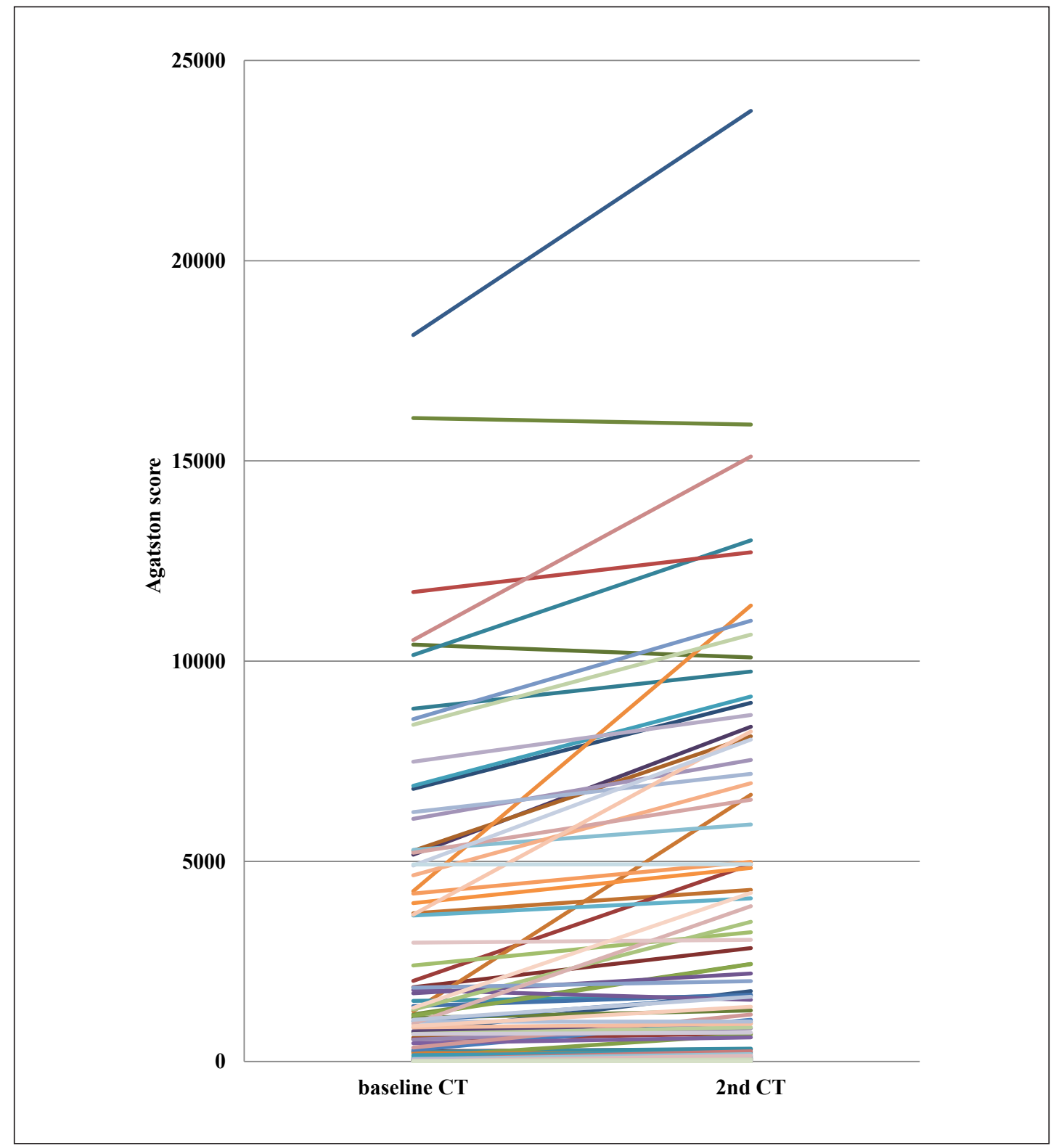

Fig. 1. Changes in the aortic calcification Agatston scores from the baseline CT to the 2nd CT scan.

Risk Factor Affecting the 2nd Agatston Scores (Table 2, Fig. 2)

After adjustment for confounders, the change in corrected serum calcium exhibited a significant non-linear correlation with the 2nd Agatston scores ( $p=0.022$ for non-linearity / $p=0.031$ for effect of corrected serum calcium) (Table 2, Fig. 2). There were no significant correlations between the other clinical parameters and the 2nd Agatston scores (Table 2).

Interaction between the Change of Calcium and the Baseline Agatston Scores (Fig. 3)

The effect of corrected serum calcium on the 2nd Agatston scores was modified by the baseline Agatston score ( $p=0.049)$, and the effect of hypercalcemia was greater in patients with higher baseline Agatston scores (Fig. 3). 


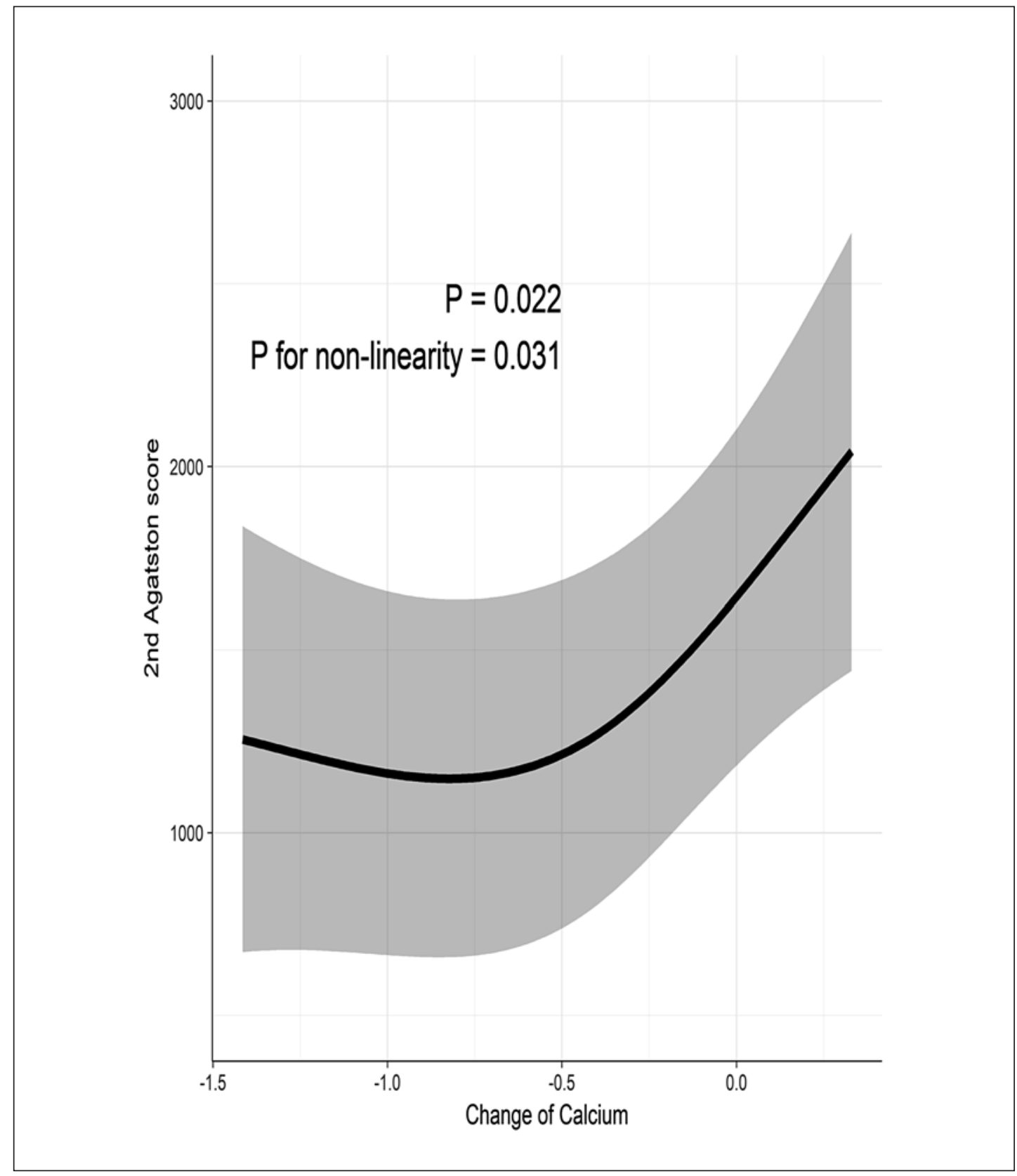

Fig. 2. The effect of the change of calcium on the 2nd Agatston score.

\section{Discussion}

In the present study, we evaluated infrarenal abdominal AoC and examined the factors associated with its advancement in KTRs. As a result, AoC was prevalent in $78.6 \%$ of KTRs and the individual Agatston scores progressed in $63.9 \%$ of KTRs (Fig. 1). AoC increased by a median of $8.2 \%$ per year in KTRs with baseline Agatston scores $>30$. After adjustment for confounders, the change in corrected serum calcium exhibited a significant non-linear correlation with the AoC (Table 2 and Fig. 2). Moreover, an interaction was evident from the 


\section{Kidney \\ Blood Pressure \\ Research}

Table 2. Risk factors affecting the 2nd Agatston scores

\begin{tabular}{l|l}
\hline Kidney Blood Press Res 2019;44:823-834 \\
\hline DOI: 10.1159/000501740 & $\begin{array}{l}\text { ○ 2019 The Author(s). Published by S. Karger AG, Basel } \\
\text { www.karger.com/kbr }\end{array}$ \\
\hline
\end{tabular}

Naganuma et al.: Aortic Calcification in Kidney Transplant Recipients

\begin{tabular}{lll}
\hline Variable (difference) & $p$ value & $\begin{array}{l}p \text { for } \\
\text { non-linearity }\end{array}$ \\
\hline Corrected calcium & & \\
Phosphate & 0.022 & 0.031 \\
Calcium $\times$ serum phosphorus product & 0.975 & 0.975 \\
i-PTH & 0.929 & 0.961 \\
Body mass index & 0.991 & 0.897 \\
eGFR & 0.12 & 0.084 \\
Calcineurin inhibitor & 0.403 & 0.268 \\
Systolic blood pressure & 0.939 & NA \\
Diastolic blood pressure & 0.583 & 0.759 \\
Total cholesterol & 0.975 & 0.973 \\
Triglycerides & 0.966 & 0.906 \\
High density lipoprotein cholesterol & 0.275 & 0.138 \\
Low density lipoprotein cholesterol & 0.512 & 0.315 \\
HbA1c & 0.854 & 0.926 \\
Serum albumin & 0.993 & 0.936 \\
C-reactive protein & 0.815 & 0.652 \\
Smoking habit & 0.759 & 0.716 \\
Use of statin & 0.299 & NA \\
\hline
\end{tabular}

i-PTH, intact parathyroid hormone; eGFR, estimated glomerular filtration rate; HbA1c, hemoglobin A1c; NA, not available.

baseline AoC in the effect of corrected serum calcium on the progression of AoC, and the effect of hypercalcemia was greater in patients with more severe VC (Fig. 3). To our knowledge, this is the first report, in which infrarenal abdominal AoC was assessed quantitatively using the Agatston score and the risk factors for the progression of AoC were investigated using nonlinear regression analysis in KTRs.

Most reported studies on VC in KTRs have investigated CAC using the Agatston score [11, 13-23], as described above, and reports on the AoC have been limited [12, 14, 16, 17]. Moreover, with respect to the evaluation of the AoC, the aortic segment measured has varied between studies and relative comparisons have been difficult. Four studies of KTRs using the Agatston score have been reported, but 2 and 1 of these examined thoracic AoC $[14,17]$ and descending AoC [16], respectively, and the remaining report examined total AoC [12]. Thus, our study initially evaluated infrarenal abdominal AoC. Studies that have evaluated aortic calcification using a semi-quantitative measurement method, such as the Aortic Calcification Index (ACI), have also occasionally been reported.

Many studies have reported the prevalence of CAC in KTRs to be $30-80 \%$; although it has varied largely between the studies $[11,13,14,17,19-22]$. With respect to the prevalence of AoC in studies using the Agatston score, Nguyen et al. [17] and DeLoach et al. [12] reported prevalences of 85 and $33.9 \%$, respectively; however, they evaluated thoracic AoC. In our study, the prevalence of AoC was 78.6\%, which was close to the value reported by Nguyen et al. [17] The frequency of AoC was lower in the study performed by DeLoach et al. [12], but the measurements were performed within 6 months after transplantation in all cases, which may explain the difference between their results and the findings of our study and those reported by Nguyen et al. [17] in which the subjects were examined years after transplantation.

Most studies on VC progression in KTRs have investigated CAC, and have concluded that progression of CAC slows down but does not halt after kidney transplant compared with dialysis patients $[11,13-15,18-23,28]$. Moreover, with the increasing length of time after transplantation, worsening of VC was also observed [11,13-15, 18-23, 28]. With respect to 


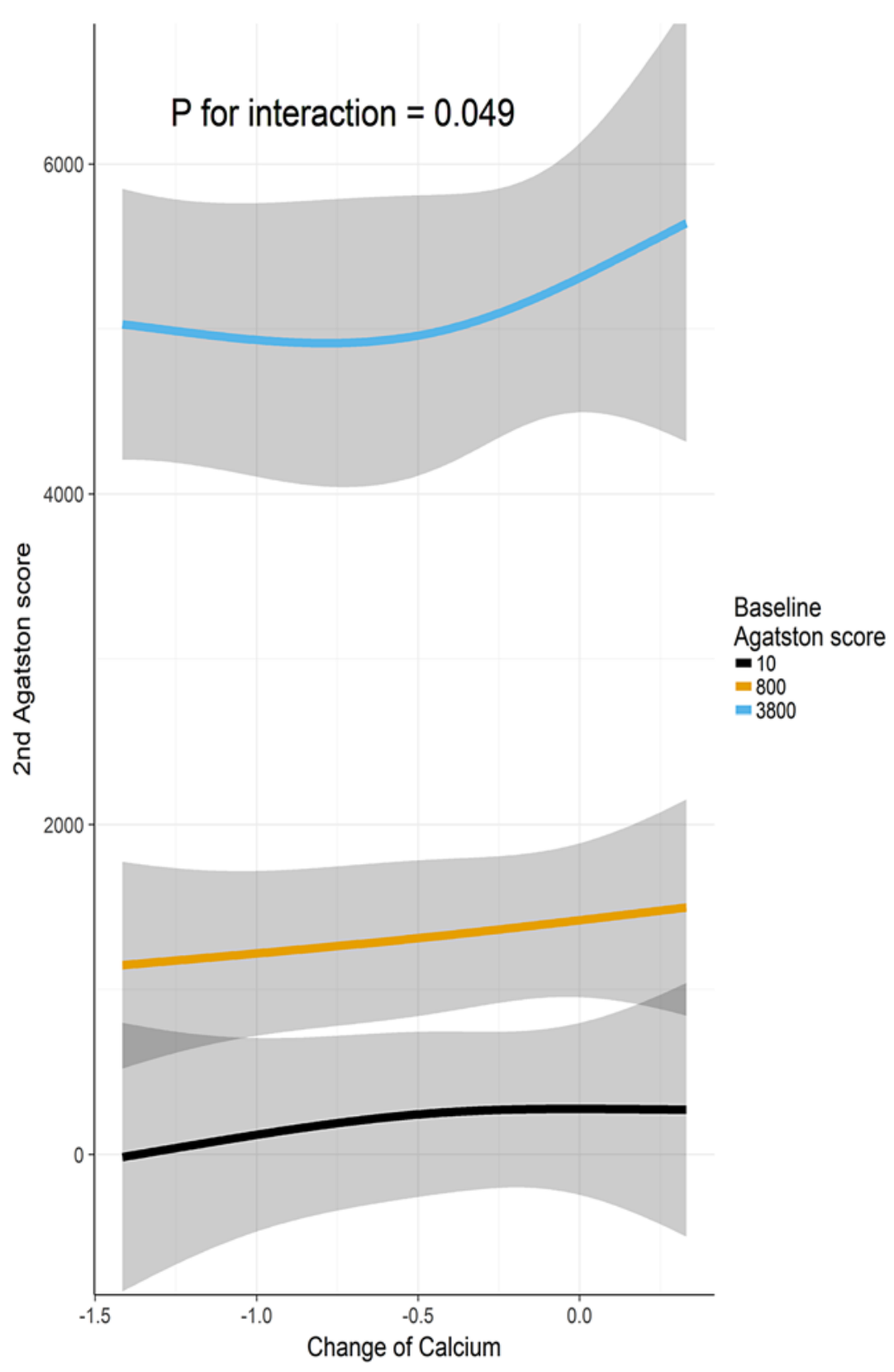

Fig. 3. Interaction analysis suggested that the effect of exposure variable (the change volume of calcium) on the 2nd Agatston score were modified by the values of baseline Agatston scores. For example, we present the effect of corrected serum calcium at 3 pattern of baseline Agatston scores (10: 25th percentile, 800: 50th percentile, and 3,600: 75th percentile).

CAC, in studies that defined progression based on the SRC method [25], which was also used by ourselves, progression was observed in $23.2-34.5 \%$ of cases [11, 13, 20-22]. However, this should be interpreted carefully because the conditions, such as the observation period and timing of the study after transplantation, varied between the studies. To our knowledge, progression of AoC was only investigated using the Agatston score in 2 previous studies (Moe 


\section{Kidney \\ Blood Pressure \\ Research}

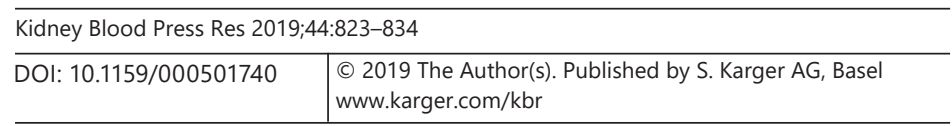

Naganuma et al.: Aortic Calcification in Kidney Transplant Recipients

et al. [16] and Maréchal et al. [14]). Moe et al. [16] showed no significant progression of AoC, which was likely a consequence of their small cohort $(n=23)$, the short follow-up period (15 to 20 months) and the timing of the investigation, which was immediately after transplantation (baseline CT scan was performed at the time of kidney transplantation). The study by Maréchal et al. [14] was the first to assess progression of AoC in a large population of KTRs ( $n=197$ ) with a relatively long follow-up (4.4 $0 \pm 0.28$ years) and time following transplantation ( $8.3 \pm 6.9$ years). They observed that AoC increased by a median of $4 \%$ per year. In our study ( $n=98$, follow-up period; 3.2 [3.0-5.1] years, time from transplantation; 5.8 [2.8-10.0] years), AoC progression was observed in $63.9 \%$ of the patients when the SRC method was used, and the annualized rate percentage was $8.2 \%$ per year, suggesting that AoC also progresses substantially in KTRs. Progression was also observed following the evaluation of AoC using ACI $[29,30]$.

The annualized rate percentage of progression of infrarenal abdominal AoC in our study was about 2 times faster than that in the study on thoracic AoC reported by Maréchal et al. [14] Maréchal et al. [14] simultaneously measured CAC and observed an annualized rate percentage of progression of $11 \%$ per year, suggesting that the calcification speed differs among regions, even in the same aorta.

Very few studies have reported risk factors of AoC progression in KTRs, and the study by Maréchal et al. [14] described above was the only one that used the Agatston score. Maréchal et al. [14] reported that baseline AoC, higher pulse pressure, statin use, older age, serum phosphate level, use of aspirin, and male sex were risk factors for AoC progression on multivariable analysis. However, since they used stepwise linear regression analysis for the multivariable analysis, it is possible that confounding factors were not adjusted for. In contrast, in our analysis, the baseline AoC, age, and sex were incorporated into the adjustment as unmodifiable factors, to adjust for confounders, and the amounts of changes in the modifiable factors were investigated as exploratory factors. In addition, non-linearity was taken into consideration in the analysis. As a result, a significant non-linear correlation with AoC progression was noted only in corrected serum calcium (Table 2 and Fig. 2). Although the study by Meneghini et al. [30] reported the evaluation of abdominal AoC using ACI, they showed that a high serum calcium level was a risk factor of AoC progression, which is consistent with our findings. While Maréchal et al. [14] extracted the serum phosphate level, higher pulse pressure, and statin therapy in their analysis, no significant differences were noted for any of these measures in our study. The reasons for the difference from our findings may be due to the location of the AoC. Reduction of the elastin/collagen ratio and elastin and collagen disarray are likely to occur in the abdominal aorta compared with those in the thoracic aorta [31,32], and this degraded elastic fiber may cause calcium deposition [33]. Another possibility is that the difference may have been due to differences in the analytical method; thus, it is not possible to draw any definitive conclusions.

Moreover, serum calcium was not found to be a risk factor of CAC progression in any of the reports on risk factors of CAC $[11,13-15,18-23,28]$. Although a combination of intimal and medial calcification may occur in patients with CKD, CAC is considered to be mainly due to intimal calcification, which is typically associated with atherosclerotic disease, and AoC to be due to medial calcification, which is mainly linked to mineral and bone disorders [34-36]. The influence of serum calcium on VC progression may readily appear in AoC due to regionassociated difference in the mechanism of VC.

Many researchers have reported the presence of baseline VC as a risk of VC progression in KTRs $[11,13,14,21,22]$. On the other hand, no development of VC is observed, even at completion of follow-up, in most cases without VC at the baseline [11, 13, 14, 21, 22]. In our patients, the 2nd Agatston score was also 0 in $71.4 \%$ of patients with a baseline Agatston score of 0 . Moreover, the effect of hypercalcemia on the progression of AoC was greater in 


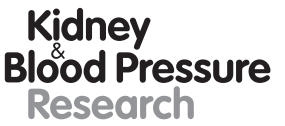

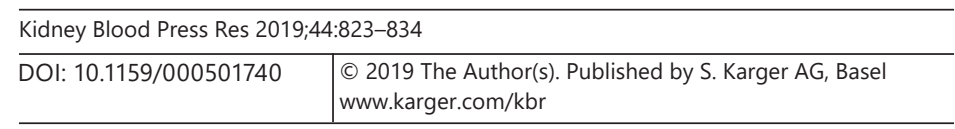

Naganuma et al.: Aortic Calcification in Kidney Transplant Recipients

patients with more severe VC (Fig. 3). These findings suggest that management of hypercalcemia is particularly important for the prevention of calcification in CKD-MBD management of KTRs with severe VC.

Egbuna et al. [37] reported that KTRs with corrected calcium $10.5 \mathrm{mg} / \mathrm{dL}$ at one year after transplantation had a significantly higher risk of graft loss compared to those without. Thus, leaving hypercalcemia untreated can be harmful and hypercalcemia has previously been used as an index for CKD-MBD management after kidney transplantation, including management of tertiary hyperparathyroidism and criteria of therapeutic intervention [38]. As shown by our findings, these may be particularly appropriate from the perspective of VC.

The strengths of our study are as follows: (1) The prevalence and progression of infrarenal abdominal AoC, risk factors, and interactions were initially investigated using the Agatston score, (2) data were collected not only at the time of inclusion but also at the 2nd CT scan to investigate risk factors and the relationship between the value of change and progression based on the Agatston score, and (3) non-linear regression analysis, in which a non-linear association was considered, was used in the analysis of the Agatston scores, thus, showing greatly distorted data distribution.

The present study also has some limitations. Firstly, not all background factors could be adjusted for, because the sample size was small, which may have reduced the statistical power. A study with a larger sample size may be necessary in the future. In addition, with respect to the duration of follow-up, investigation for a longer follow-up period may be necessary. Secondly, only KTRs were investigated with no control group. A comparison of the frequency of AoC and risk factors between healthy subjects and CKD patients will be necessary. Thirdly, it is desirable to follow all patients from the initiation of kidney transplantation, because the risks of $\mathrm{VC}$ and arteriosclerosis vary depending on the time after kidney transplantation (e.g. due to the amount of immunosuppressive agents). Fourthly, we only investigated the infrarenal abdominal AoC. However, the relationships with CAC and AoC in other regions and differences in risk factors should be investigated.

In conclusion, the present study revealed that hypercalcemia was a risk factor for the development of infrarenal abdominal AoC in kidney transplantation recipients. Furthermore, the effect of hypercalcemia was greater in patients with more severe VC. Further studies are needed to explore whether infrarenal abdominal AoC predicts cardiovascular events and allcause mortality in KTRs.

\section{Acknowledgements}

We thank the participants for providing us their clinical information.

\section{Statement of Ethics}

This study protocol was conducted in accordance with the Principles of the Declaration of Helsinki and the Declaration of Istanbul, and was approved by the ethics committee of the Osaka City University Graduate School of Medicine (No. 1415).

\section{Disclosure Statement}

The authors declare that they have no competing interests. 


\section{Kidney \\ Blood Pressure Research}

\begin{tabular}{l|l}
\hline Kidney Blood Press Res 2019;44:823-834 \\
\hline DOI: 10.1159/000501740 & $\begin{array}{l}\text { @ 2019 The Author(s). Published by S. Karger AG, Basel } \\
\text { www.karger.com/kbr }\end{array}$ \\
\hline
\end{tabular}

Naganuma et al.: Aortic Calcification in Kidney Transplant Recipients

\section{Funding Sources}

This work was supported by grants from the Osaka City University Medical Research Foundation.

\section{Author Contributions}

T.N. (corresponding author) contributed to the concept, design, data acquisition, interpretation, writing. D.K. and A.S. contributed to the data analysis and interpretation. Y.T., U.J. and T.N. reviewed and revised the manuscript. All authors read and approved the final manuscript.

\section{References}

1 Kahwaji J, Bunnapradist S, Hsu JW, Idroos ML, Dudek R. Cause of death with graft function among renal transplant recipients in an integrated healthcare system. Transplantation. 2011 Jan;91(2):225-30.

2 Ojo AO, Morales JM, González-Molina M, Steffick DE, Luan FL, Merion RM, et al.; Scientific Registry of Transplant Recipients and; Spanish Chronic Allograft Study Group. Comparison of the long-term outcomes of kidney transplantation: USA versus Spain. Nephrol Dial Transplant. 2013 Jan;28(1):213-20.

3 Budoff MJ, Nasir K, Katz R, Takasu J, Carr JJ, Wong ND, et al. Thoracic aortic calcification and coronary heart disease events: the multi-ethnic study of atherosclerosis (MESA). Atherosclerosis. 2011 Mar;215(1):196-202.

4 Budoff MJ, Shaw LJ, Liu ST, Weinstein SR, Mosler TP, Tseng PH, et al. Long-term prognosis associated with coronary calcification: observations from a registry of 25,253 patients. J Am Coll Cardiol. 2007 May;49(18): 1860-70.

5 Eisen A, Tenenbaum A, Koren-Morag N, Tanne D, Shemesh J, Imazio M, et al. Calcification of the thoracic aorta as detected by spiral computed tomography among stable angina pectoris patients: association with cardiovascular events and death. Circulation. 2008 Sep;118(13):1328-34.

6 Kondos GT, Hoff JA, Sevrukov A, Daviglus ML, Garside DB, Devries SS, et al. Electron-beam tomography coronary artery calcium and cardiac events: a 37-month follow-up of 5635 initially asymptomatic low- to intermediate-risk adults. Circulation. 2003 May;107(20):2571-6.

7 Blacher J, Guerin AP, Pannier B, Marchais SJ, London GM. Arterial calcifications, arterial stiffness, and cardiovascular risk in end-stage renal disease. Hypertension. 2001 Oct;38(4):938-42.

8 London GM. Cardiovascular calcifications in uremic patients: clinical impact on cardiovascular function. J Am Soc Nephrol. 2003 Sep;14(9 Suppl 4):S305-9.

9 Honkanen E, Kauppila L, Wikström B, Rensma PL, Krzesinski JM, Aasarod K, et al.; CORD study group. Abdominal aortic calcification in dialysis patients: results of the CORD study. Nephrol Dial Transplant. 2008 Dec;23(12):4009-15.

10 Matsuoka M, Iseki K, Tamashiro M, Fujimoto N, Higa N, Touma T, et al. Impact of high coronary artery calcification score (CACS) on survival in patients on chronic hemodialysis. Clin Exp Nephrol. 2004 Mar;8(1):54-8.

11 Bargnoux AS, Dupuy AM, Garrigue V, Jaussent I, Gahide G, Badiou S, et al. Evolution of coronary artery calcifications following kidney transplantation: relationship with osteoprotegerin levels. Am J Transplant. 2009 Nov; 9(11):2571-9.

12 DeLoach SS, Joffe MM, Mai X, Goral S, Rosas SE. Aortic calcification predicts cardiovascular events and all-cause mortality in renal transplantation. Nephrol Dial Transplant. 2009 Apr;24(4):1314-9.

13 Gulcicek S, Zoccali C, Olgun DC, Tripepi G, Alagoz S, Yalın SF, et al. Long-Term Progression of Coronary Artery Calcification Is Independent of Classical Risk Factors, C-Reactive Protein, and Parathyroid Hormone in Renal Transplant Patients. Cardiorenal Med. 2017 Oct;7(4):284-94.

14 Maréchal C, Coche E, Goffin E, Dragean A, Schlieper G, Nguyen P, et al. Progression of coronary artery calcification and thoracic aorta calcification in kidney transplant recipients. Am J Kidney Dis. 2012 Feb;59(2):25869.

15 Mazzaferro S, Pasquali M, Taggi F, Baldinelli M, Conte C, Muci ML, et al. Progression of coronary artery calcification in renal transplantation and the role of secondary hyperparathyroidism and inflammation. Clin J Am Soc Nephrol. 2009 Mar;4(3):685-90.

16 Moe SM, O’Neill KD, Reslerova M, Fineberg N, Persohn S, Meyer CA. Natural history of vascular calcification in dialysis and transplant patients. Nephrol Dial Transplant. 2004 Sep;19(9):2387-93.

17 Nguyen PT, Coche E, Goffin E, Beguin C, Vlassenbroek A, Devuyst O, et al. Prevalence and determinants of coronary and aortic calcifications assessed by chest CT in renal transplant recipients. Am J Nephrol. 2007; 27(4):329-35. 
18 Nguyen PT, Henrard S, Coche E, Goffin E, Devuyst O, Jadoul M. Coronary artery calcification: a strong predictor of cardiovascular events in renal transplant recipients. Nephrol Dial Transplant. 2010 Nov;25(11):3773-8.

19 Oschatz E, Benesch T, Kodras K, Hoffmann U, Haas M. Changes of coronary calcification after kidney transplantation. Am J Kidney Dis. 2006 Aug;48(2):307-13.

20 Roe P, Wolfe M, Joffe M, Rosas SE. Inflammation, coronary artery calcification and cardiovascular events in incident renal transplant recipients. Atherosclerosis. 2010 0ct;212(2):589-94.

21 Schankel K, Robinson J, Bloom RD, Guerra C, Rader D, Joffe M, et al. Determinants of coronary artery calcification progression in renal transplant recipients. Am J Transplant. 2007 Sep;7(9):2158-64.

22 Seyahi N, Cebi D, Altiparmak MR, Akman C, Ataman R, Pekmezci S, et al. Progression of coronary artery calcification in renal transplant recipients. Nephrol Dial Transplant. 2012 May;27(5):2101-7.

23 Yazbek DC, de Carvalho AB, Barros CS, Medina Pestana JO, Canziani ME. Effect of Statins on the Progression of Coronary Calcification in Kidney Transplant Recipients. PLoS One. 2016 Apr;11(4):e0151797.

24 Ichii M, Ishimura E, Shima H, Ohno Y, Ochi A, Nakatani S, et al. Quantitative analysis of abdominal aortic calcification in CKD patients without dialysis therapy by use of the Agatston score. Kidney Blood Press Res. 2013; 38(2-3):196-204.

25 Hokanson JE, MacKenzie T, Kinney G, Snell-Bergeon JK, Dabelea D, Ehrlich J, et al. Evaluating changes in coronary artery calcium: an analytic method that accounts for interscan variability. AJR Am J Roentgenol. 2004 May;182(5):1327-32.

26 Block GA, Spiegel DM, Ehrlich J, Mehta R, Lindbergh J, Dreisbach A, et al. Effects of sevelamer and calcium on coronary artery calcification in patients new to hemodialysis. Kidney Int. 2005 Oct;68(4):1815-24.

27 Expert Committee on the Diagnosis and Classification of Diabetes Mellitus. Report of the expert committee on the diagnosis and classification of diabetes mellitus. Diabetes Care. 2003 Jan;26 Suppl 1:S5-20.

28 Cianciolo G, Capelli I, Angelini ML, Valentini C, Baraldi O, Scolari MP, et al. Importance of vascular calcification in kidney transplant recipients. Am J Nephrol. 2014;39(5):418-26.

29 Imanishi K, Hatakeyama S, Yamamoto H, Okamoto A, Imai A, Yoneyama T, et al. Post-transplant renal function and cardiovascular events are closely associated with the aortic calcification index in renal transplant recipients. Transplant Proc. 2014;46(2):484-8.

30 Meneghini M, Regalia A, Alfieri C, Barretta F, Croci D, Gandolfo MT, et al. Calcium and osteoprotegerin levels predict the progression of the abdominal aortic calcifications after kidney transplantation. Transplantation. 2013 Jul;96(1):42-8.

31 Lindesay G, Bézie Y, Ragonnet C, Duchatelle V, Dharmasena C, Villeneuve N, et al. Differential Stiffening between the Abdominal and Thoracic Aorta: Effect of Salt Loading in Stroke-Prone Hypertensive Rats. J Vasc Res. 2018;55(3):144-58.

32 Zhang J, Zhao X, Vatner DE, McNulty T, Bishop S, Sun Z, et al. Extracellular Matrix Disarray as a Mechanism for Greater Abdominal Versus Thoracic Aortic Stiffness With Aging in Primates. Arterioscler Thromb Vasc Biol. 2016 Apr;36(4):700-6.

33 Atkinson J. Age-related medial elastocalcinosis in arteries: mechanisms, animal models, and physiological consequences. J Appl Physiol (1985). 2008 Nov; 105(5):1643-51.

34 London GM, Marchais SJ, Guerin AP, Metivier F, Adda H. Arterial structure and function in end-stage renal disease. Nephrol Dial Transplant. 2002 Oct;17(10):1713-24.

35 Moe SM, Chen NX. Mechanisms of vascular calcification in chronic kidney disease. J Am Soc Nephrol. 2008 Feb; 19(2):213-6.

36 Schoppet M, Shroff RC, Hofbauer LC, Shanahan CM. Exploring the biology of vascular calcification in chronic kidney disease: what's circulating? Kidney Int. 2008 Feb;73(4):384-90.

37 Egbuna OI, Taylor JG, Bushinsky DA, Zand MS. Elevated calcium phosphate product after renal transplantation is a risk factor for graft failure. Clin Transplant. 2007 Jul-Aug;21(4):558-66.

38 Fukagawa M, Yokoyama K, Koiwa F, Taniguchi M, Shoji T, Kazama JJ, et al.; CKD-MBD Guideline Working Group; Japanese Society for Dialysis Therapy. Clinical practice guideline for the management of chronic kidney disease-mineral and bone disorder. Ther Apher Dial. 2013 Jun;17(3):247-88. 